\title{
Schmiedebergs Werk.
}

Von

\author{
Hans H. Heyer.
}

In seinem 83. Lebensjahr, in fast unveränderter geistiger Lebendigkeit und Schaffenslust ist 0 . Schmiedeberg nach kurzer Krankheit am 12. Juli 1921 verschieden. Mit ihm hat die pharmakologische Wissenschaft nicht nur ihr allgemein anerkanntes Haupt, sondern zugleich ihren eigentlichen Schöpfer und Banmeister verloren.

Dem ehrenden Nachruf, den B. Naunyn im 90. Bande dieses Archivs dem langjährigen Freunde und Arbeitsgenossen gewidmet hat, will ich als Schmiedebergs Schüler den bescheidenen Versuch einer Würdigung seiner wissenschaftlichen Persönlichkeit und seines Werkes folgen lassen.

Oswald Schmiedeberg ward als Sohn eines Forstmannes in Kurland am 11. Oktober 1838 geboren, verbrachte nach der Eltern Übersiedelung nach Esthland seine Jugend in Dorpat und widmete sich dort nach beendeter Gymnasialzeit dem Studium der Medizin. Am 16. April 1866 ward er zum Doktor promoviert auf Grund einer unter Buchheims Leitung ausgefuhrten Arbeit über Bestimmung nnd Verhalten des Chloroform im Blut, einer scharfsinnigen und methodisch eingehenden Experimentalkritik der bis dahin allein maßgebenden Versuche von Lallemand, Perrin und Duroy ïber das Verbalten der Anästhetica im Organismus (1860). Die Arbeit läßt bereits den kenntnisreichen, griundlichen und peinlich gewissenhaften Forscher erkennen. Kurz darauf wurde Sehmiedeberg Assistent am Pharmakologischen Institut, nach zwei weiteren Jahren, 1868, Privatdozent und im Mai 1869, als sein Lehrer Buchheim an die Universität Gießen war berufen worden, zu dessen Nachfolger ernannt als Professor der Pharmakologie, Diätetik und Geschichte der Medizin.

Neben Buchbeim waren es namentlich der Chemiker Karl Schmidt und der Physiologe Friedrich Bidder - beide berühmt durch ihre gemeinsame grundlegende Arbeit über die epidemische 
Cholera - sowie in Leipzig Karl Ladwig, die auf Schmiedebergs wissenschaftliche Entwicklung und Ausbildung eingewirkt haben; dén gleich nach seiner Ernennung zum Professor hatte Schmiedeberg einen einjährigen Urlaub genommen, um in Karl Ludwigs einzigartiger Schule die Meisterschaft des physiologischen Experimentierens zu erlangen. In Leipzig trat er auch in einen größeren Kreis hervorragender jüngerer Gelehrter wie u. a. Böhm, Bowditch, Hüfner, Miescher, mit denen ihn in der Folge dauernde. Freundschaft verbunden hat.

Nach Dorpat zuriuckgekehrt hat Schmiedeberg dort nur noch kurze Zeit gewirkt; indes war es für ihn persönlich wie für die spätere Entwickelung seiner wissenschaftlichen Bestrebungen von großer Bedeutung, daB er in seiner Dorpater Dozentenzeit mit dem gleichzeitig dort ornannten Professor der inneren Medizin, Bernhard Naunyn, in nahe Beziehung trat; sie hat später zur Gründung dieses Archivs geführt als einer fachmännisch geleiteten und alsbalä in der ganzen medizinischen Welt als maßgebend anerkannten Sammelstätte der experimentell-pharmakologischen Forschung. Als 1872 die Deutsche Universität in Straßburg gegrïndet wurde, ward unter den hervorragendsten deutschen Gelehrten, die der Universitätskurator Freiberr von Roggenbach nach Straßburg zog, auf K. Ludwigs glückliche Empfehlung auch Schmiedeberg hinberufen: hier ist er als der Einzige ihrer Gründer bis zur Auflösung und Vertreibung der deutschen Hochschule durch die Franzosen tätig geblieben.

Als äußeres Denkmal seines großartigen Wirkens in Straßbarge hat er das nach seinen bis in alle Einzelheiten durchdachten und genauen Angaben ron dem Architekten Prof. Warth erbaute pharmakologische Institut hinterlassen, das nach seiner zweckvollen Anordnong und Einrichtung wie anch nach seiner formenschönen Gestaltung alle Bewunderung verdient. Eine von Schmiedeberg selbst verfaßte Beschreibung nebst Grundriß und Abbildung findet sich in dem Werk von S. Hansmann $\gg$ Die Kaiser Wilhelm Universitäs Straßburg * Straßburg 1897.

Bis zur Fertigstellung dieses Prachtbaus im Jahre 1887, also die ersten entscheidenden 15 Jahre, war das pharmakologische Institut in den sehr bescheidenen, immerhin fürs erste leidlich zureichenden Räumen des oberen Stockwerks der ehemaligen École de médecine an der Metzgergießen untergebracht. In den unteren Geschossen lagen die Institate von Goltz und von Hoppe-Seyler, an der Straße gegenüber die etwas schäbige, kleinbürgerliche Speisewirtschaft >zum Rindsfuß , welche uns Assistenten und Laboranten der 
drei Institute bei den Mahlzeiten zu einer mitteilsamen, lebhaft einander anregenden und arbeitsfrohen Gesellschaft vereinigte.

Um die Zeit des Straßburger Anfangs war die Pharmakologie noch ein fast nirgend sonst gepflegter, ja kaum gekannter und beachteter, oft wirklich auch vertrockneter Zweig der medizinischen Wissenschaft: man braucht sich nur jenes geringschätzigen Urteils zu erinnern, welches Billroth noch 1876 in seiner bekannten Schrift „Über Lehren und Lernen der medizinischen Wissenschaften an den Universitäten deutseher Nation * über die sogenannte Pharmakologie and ihre Rolle im medizinischen Unterricht, nicht ohne einen starken Schein von Recht, äußern durfte. Und doch hatte schon 30 Jahre lang ein hervorragender Gelehrter an der Um- und Neubildnng der alten Pharmakologie zielbewaBt und unermüdlich gearbeitet und tatsächlich anch bereits den planvoll entworfenen Grundstock einer experimentell-biologischen Arzneiwissenschaft als eines Teiles der allgemeinen Physiologie angelegt: das war Rudolf Buchheim. Schmiedeberg selbst hat seines Lehrers große und entscheidende Bedentung fïr die Nenschaffung der wissenschaftlichen Pharmakologie in eingehender Besprechnng im 97. Bande dieses Arehirs (1912) gewürdigt and auch die Grinde dargelegt, warum sein einsames Werk trotz mancher warmen und verständnisvollen Anerkennungen im ganzen anbekannt und in den Kreisen der Ärzte und medizinischen Fakultåten unverstanden blieb.

Das von Buchheim 1847 gegründete experimentell-pharmakologische Institut in Dorpat war das erste und einzige seiner Art and blieb es noch viele Jahre lang; und erst seit den 70er Jahren wurden nach und nach an den deutschen Hochschnlen solche Laboratorien eingerichtet, so daß Schmie deberg 1912 sehreiben konnte: „Gegenwärtig fehli zwar an keiner dentschen Universität ein derartiges Institut, aber meist sind sie in Räumen untergebracht, die für andere Institute unbrauchbar geworden waren. « Heute trifft auch diese Einschränkung kaum mehr zu. Gegen vierzig von den pharmakologischen Lehrstiuhlen des In- und Auslandes waren oder sind gegenwärtig besetzt von seinen unmittelbaren Schülern - ein Erfolg wie ihn von Lehrern eines theoretischen Faches der Medizin vielleicht nur noch K. Ludwig oder R. Koch erlebt haben. Diese rasche und fruchtbare Entwicklung der Pharmakologie als Wissenschaft und als Lehrfach ist zugleich ein Beweis von der allgemein bei den medizinischen Fakultäten durchgedrungenen Einsicht in die Bedeutung und Unentbehrlichkeit pharmakologischer Forschung und Lehre, und von dem erwachten Bedürfnis nach einem physiologischen Verständnis der 
Arzneiwirkungen sowie experimenteller Begründung ihrer Verwendung. Diese Errungenschaft aber ist zum großen Teil das Werk Schmiedebergs.

Schmiedebergs Laboratorium in StraBburg ward bald der Sammelpunkt zahlreicher junger Gelehrten, die ihm aus dem In- und Ausland zuströmten: sie bildeten die Pioniertruppe, die unter seiner Fübrung die weiten, noch unbebauten Gebiete der Pharmakologie erschließen half. Die Frucht dieser gemeinsamen Arbeit ist in über 200 Abhandlungen fast sämtlich in diesem Archiv niedergelegt worden.

Das Wichtigste davon sei im Folgenden angeführt:

Schon jene erste wissenschaftliche Arbeit Schmiedebergs über das Verhalten des Chloroforms im Blut gab AnstoB zu einer ganzen Reihe weiterer Untersuchungen Schmiedebergs und seiner Schtiler uber die Pharmakologie des Chloroforms und der ihm verwandten Stoffe aus der »Gruppe des Alkohols«. Als besonders bedeutsam hebe ich daraus die Arbeit Sehmiedebergs hervor über die pharmakologisehen Wirkungen und die therapeutische Anwendung einiger Carbaminsäureester ${ }^{1}$ ); Schmiedeberg behandelt darin die grundsätzliche Frage nach der Abhängigkeit der narkotischen Wirksamkeit der betreffenden Verbindungen von ihrem chemischen Aufban, indem er den einzelnen Atomgruppen, wie den aliphatischen Kohlenwasserstoffgruppen und den jeweils damit verbundenen sonstigen Gruppen, einem Säureradikal oder einem Ammoniakrest, ihre besondere Einwirkung innerhalb der Gesamtwirkung des Stoffes zuteilt. Dieser Vorstellung entsprang die von Schmiedeberg unmittelbar gegebene und z. T. von ihm selbst befolgte Anregung zur experimentellen Prifung und klinischen Einfuhrung des Paraldebyds, des Amylenhydrats und vor allem des Urethans, welches dann die später folgende große Reihe hypnotisch wirkender Harnstoffverbindungen nach sich zog. Schmiedebergs Auffassung ist lange Zeit allein herrschend geblieben und hat außer zu zahllosen meist freilich verfehlten Versuchen zur synthetischen Gewinnung ron Arzneimitteln mit vorausberechneten Wirkungen, zu vielen Experimentalarbeiten and hypothesenreichen Abhandlungen und selbst zu umfangreichen Werken solcher Art den Anlaß gegeben. Auch die Ehrlichsche Hypothese von den haptophoren und toxophoren Gruppen geht auf analoge Vorstellungen zuriuck. Gegenwärtig scheint die Annahme, daB die Atomgruppen einer organischen Verbindung als solche in unmittelbare »molekulare* Wechselwirkung mit den Körperzellbestand-

1) Dieses Archiv 1885, Bd. 20. 


\section{$-\mathrm{V}-$}

teilen treten und dadurch jede in ihrer spezifischen Weise die Wirkungsart der ganzen Verbindung bestimmen, der mehr und mehr begrindeten Einsicht weichen zu wollen, daß die primären pharmakologischen Wirkungen solcher Stoffe sich in der Regel nicht sowohl additiv aus den, ihren verschiedenen Atomgruppen mit mehr oder weniger Begründung zugeschriebenen Einzelwirkungen zusammensetzen, als vielmehr durch den einheitlichen Gesamtcharakter des Stoffes, d. h. Konstitutiv bestimmt werden. Daß freilich die skonstitutiven " Eigenschaften einer Verbindung im letzten Grunde von der Art der in ihr vereinigten Atomgruppen mit bedingt sind, versteht sich von selbst, and jusofern müssen, wie sehon W. Ostwald hervorgehoben hat, grundsätzlich anch sie anf die additive Form sich zurückführen lassen. Tatsächlich ist es aber bisher nur in einigen wenigen Fällen, z. B. in gewissen Reihen homologer Verbindungen gelungen, einen bestimmten and meßbaren konstitutiven Einfluß der Atomgruppen und damit eine erkennbare Abhängigkeit der pharmakologischen Wirkung von ihnen aufzufinden oder vorauszusagen.

Eine andere Reihe grundsätzlich wichtiger Forschungen und Fortschritte in der Pharmakologie hat ihren Ausgang genommen von der klassischen Arbeit Schmiedebergs iber das Gift des Fliegenpilzes1) 1869 und die daran anknüpfende Untersuchung über die Nikotinwirkung am Herzen 2). Die Muskarinarbeit ist ein untibertroffenes Muster einer vollständigen pharmakologischen Drogenuntersuchung, sowohl in bezug auf die chemische Auffindung, Darstellung und Analyse des wirksamen Stoffes, wie in der systematisohen und kritischen Experimentaluntersuchung seiner Wirkungen. Außer dem in solcher Art bis dahin uberhaupt ganz unbekannten, toxikologiseb and therapeutisch wichtigen wechselseitigen Antagonismus zwischen Muskarin und Atropin, sowie dem einseitigen zwischen Muskarin und Nikotin, haben diese Untersuchungen auch die Kenntnis von der Herzinnervation and insbesondere dem vagalen Hemmungsapparat gefördert und das Vorhandensein eines nikotinempfindlichen Zwischenstiickes zwischen Vagusnerv und seiner letzten Endigung erwiesen des später als gangliöse Synapse erkannten Gebildes. Auch die wichtige Entdeckung der mit dem Vagusstamm verbundenen Akzeleransfasern beim Frosch, die lange Latenz und Nachwirkung nach ihrer Reizung, ist ans der Nikotinuntersuchung hervorgegangen. Die ganze Pharmakologie der vegetativen Organsysteme, der hearistisch

1) Das Mnskarin, Leipzig, Vogel 1869.

2) Truhart, Diss, Dorpat 1869; $\$$ chmiedeberg, Ber. Kgl. Sächs. Ges. d. W. 1870. 
und didaktisch unentbehrliche Schematismus der $\gg$ Systemgifte (der parasympathischen, sympathischen usw. Gifte) gehen auf diese ersten Arbeiten Schmiedebergs zurück.

Eine andere, der Muskarinarbeit in der Schwierigkeit des Problems wie in seiner meisterhaften methodischen und kritischen Behandlung, endlich auch in ihrer wissenschaftlichen und praktischen Tragweite ebenbuirtige Untersuchung betrifft die Darstellung der wirksamen Digitalisstoffe und die experimentelle Analyse ihrer Wirkungen auf die Kreislauforgane ${ }^{1}$ ). Sch miedebergs chemische, durch die pharmakologische Priffung schrittweise geleitete Untersuchung der Digitalisbestandteile lieferte die erste wichtige und bis heute noch in ihren wesentlichen Teilen baltbare Grundlage der uberaus schwierigen Digitalischemie, auf der alle späteren Untersucher weitergearbeitet baben. Für das bis dahin noch ganz unklare und vielfach umstrittene Verständnis von der Wirkungsart der Digitalis und ihrer therapeutischen Verwendbarkeit war Schmiedebergs Analyse ihrer Herzwirkungen von entscheidender Bedeutung; durch sie erst ward die von manchen alten Beobachtern wohl vermutete aber durch nichts bewiesene und von der Mehrzahl der Ärzte sogar gelengnete günstige Wirkung der Digitalis anf die Herzleistung klar erkannt und experimentell erwiesen. Eine zusammenfassende, auch historisch geschmückte Abhandlung ïber die Chemie und Pharmakologie der ganzen "Gruppe des Digitalins", insbesondere der Oleander- und der Digitalis-Glykoside hat Sch mied eberg im 16. Bande dieses Archivs gegeben (1883). Mehrfache Ergänzungen sind dann später durch die Arbeiten seiner Schüler hinzugekommen.

Als pharmakologisch im engeren Sinne sind ferner Schmiedebergs Untersuchungen und die daran anschließenden Arbeiten seiner Schüler über die Wirkungen des Koffeins und verwandter Purink örper gebührend hervorzuheben; sie nahmen ibren Ausgangspunkt von einer unter Schmiedebergs Leitung ausgeführten Dorpater Dissertation von 0. Johannsen (1869), durch die die eigenartige unmittelbar kontraktionsfördernde Wirkung des Kofleins auf die gestreifte Muskulatur zum erstenmal richtig erkannt und gedentet, und die irrtumliche Auffassung fruherer Beobachter widerlegt wurde, wonach das Koffein die Muskeln lediglich wie Strychnin vom Zentralnervensystem aus in Krampf versetze. Die früheren und auch noch später sich geltend machenden Widersprüche in den Beobachtungen

1) Schmied eberg, Dieses Archiv 1874, Bd. 3; Ludwigs Festschrift Leipzig 1875; Fr. Williams, Dieses Archiv 1880, Bd. 13. Dahingehörig auch Schmiedeberg über die elastische Verkiirzung usw., Dieses Archiv. 1917, Bd..82. 
der Autoren klärte Schmiedeberg durch die bemerkenswerte Entdeckung auf, daß die beiden Froscharten, R. esculenta und R. temporaria zwar nicht grundsätzlich aber doch quantitativ verschieđen mit ihren Organen auf das Koffein ansprechen, daß bei der $\mathbb{R}$. esculenta das Rückenmark, bei der R. temporaria der Muskel empfänglicher fur die Giftwirkung ist1). Auch die Koffeindiurese hat Schmiedeberg untersucht, und auf seine Anregung ist sie von W. v. Schröder experimentell analysiert ma im wesentlichen aufgeklärt worden 2 ).

Außer den hier angeftuhrten, von ihm zum größten Teil selbst behandelten Fragen der Pharmakologie hat Schmiedeberg durch die groBe Zahl seiner Schüler nahezu alle wichtigen pharmakologischen Probleme bearbeiten lassen. Neben der Untersuchung sehr zahlreicher, ja der meisten theoretisch oder praktisch wichtigen organischen Arzneistoffe und Gifte mit Einschlub der tierischen Gifte, bildete ein sehr umfangreiches Arbeitsgebiet u. a. die systematische Untersuchung der akuten Wirkungen der Schwermetalle. Die älteren toxikologischen und pharmakologisehen Untersuchungen darüber waren widersprechend und fehlerhaft, weil sie die ortlichen von den resorptiven Wirkungen zu trennen und zu unterscheiden nicht verstanden hatten. Durch die von Schmiedeberg geulbte Anwendung komplexer bzw. organischer Metallverbindungen gelang es, die örtlichen, auf unmittelbarer Eiweißbindung beruhenden Metallsalzwirkungen auszuschließen und die Fernwirkungen auf Nerv, Muskel, Kreislauf und Stoffwechsel zur Anschaung zu bringen.

Die Früchte all dieser Arbeiten hat Schmiedeberg in seinem klassisch gewordenen *Grundriß der Arzneimittellehre" 1883 gesichtet und für den ihm vorschwebenden Zweck verarbeitet, den Zweck, "die Wirkungen der in praktiseher Richtung wichtigen Agentien namentlich in bezug auf den Menschen zu schildern, die Folgen, die sich nach der Anwendung solcher Mittel unter verschiedenen Bedingungen für den Gesamtorganismus ergeben, za charakterisieren und aus den pharmakologischen Tatsachen die allgemeinen Regeln für den Gebrauch der Arzneimittel abzuleiten*. Mit fast zu peinlicher Gewissenhaftigkeit hatte Schmiedeberg vermieden, dem Werk den umfassenderen Titel einer "Pharmakologie zu geben, weil er letztere verstanden wissen wollte als die $»$ Lehre von den im lebenden Organismus durch chemisch wirkende Substanzen hervorgebrachten Veränderungen im allgemeinen, ohne Rücksicht darauf, ob sie für Heil-

1) Dieses Arehiv 1874, Bd. 2; Ber. d. D. chem. Ges. 1901, Bd. 34.

2) Dieses Archiv 1886, Bd. 22, 1887, Bd. 24; Schmiedeberg, D. Arch. f. klin. Med. 1905, Bd. 82. 
zwecke gebrancht werden oder nicht", die Arzneimittellehre aber es nur mit solehen Agentien und Wirkungen zu tun habe, die zur Heilung von Krankheiten dienen mögen. Erst in den späteren Auflagen erhielt das Buch die Bezeichnung »Grundriß der Pharmakologie in Bezug auf Arzneimittellehre und Toxikologie «. In der bedeutsamen Einleitung des Werkes entwickelt Schmiedeberg seine grundsätzlichen, in der Folge allgemein angenommenen und erweiterten Anschauungen von der Art der pharmakologischen Wirkangen; unter dem Begriff der chemisch-pharmakologischen Wirkung versteht er nicht nur im engeren Sinne chemische, sondern vor allen Dingen auch "molekulare Vorgänge, wie sie uns zum Beispiel bei der Bildung der Lösungen entgegentreten, d. h. also Vorgänge, »welche in das Gebiet der physikalischen Chemie fallen". Der Abschnitt sdie Quellen der Arzneimittellehre " bildet eine kurze aber um so eindrucksvollere Skizze ihres unerfreulichen und muihsamen Entwicklungsganges, der Abschnitt ibber » Einteilung der pharmakologisehen Agentien und der Arzneimittel $\ll$ bespricht den Wert und die Unentbehrlichkeit einer nach Buchheims Vorgang (1856) durchgefübrten Systematik, d. h. Zusammenfassung der in ihren Grundwirkungen und pharmakologiseh bedeutsamen Eigenschaften einander ähnlichen Stoffe zu Gruppen eines $*$ natürlichen Systems«, ähnlich wie etwa in der Botanik das natürliche System der Pflanzenfamilien. Für die Zwecke einer praktischen Arzneimittellehre, die nur gewisse ärztlich verwertbare Wirkungen mit ihren Folgen berïcksichtigen will, ist ein solches System nicht durchgehends verwendbar, wohl aber möglich und notwendig für Zwecke der Forsehung; denn *Untersuchungen, die ohne Berticksichtigung einer solchen Systematik ausgeführt sind, haben gegenwärtig kaum einen Wert*.

Schmiedebergs Grundriß ist in die meisten Kultursprachen iibersetzt worden und hat noch kurz vor dem Krieg die siebente Auflage erlebt. Das ist um so bemerkenswerter, als es sich keineswegs um ein leicht und mühelos genießbares Werk handelt: sein strenger, knapper Stil, der eine ungemein große Fillle nicht nur von Tatsachen, sondern auch von eingehend kritischen Erörterungen in gedrängter Kürze darbietet, verlangt vielmehr die volle Aufmerksamkeit und ernste Hingabe des Lesers, um den gedankenreichen Inhalt zu erfassen und anszuschöpfen. Dem Denkenden aber gibt dieses Meisterwerk die wissenschaftlich gesicherten und analysierten Beobachtungen der experimentellen Pharmakologie in verständlicher, organisch gegliederter und Richtung weisender Darstellung; es bildet zugleich die beste Widerlegung des Vorwurfs, der gegen Schmiede- 
bergs Schale eine Zeitlang ist erhoben worden, als kutmmere sie sich nicht um die Forderungen der praktischen Medizin und lehne die Beziehung zur Therapie grundsätzlich ab: im Gegenteil, diese notwendige Beziehung wird ron Schmiedeberg ansdrücklich hervorgehoben und in vielen erklärenden Hinweisen für die Prazis nutzbar gemacht. Allerdings stets nur in grundsätzliehen Regeln, aus denen für den einzelnen Krankheitsfall Nutzen zu ziehen, Sache des denkenden Arztes bleibt, »die Pharmakologie ist ein Wegweiser fuir die Therapie; welchen Weg diese einsehlagen will, hat sie selber za entscheiden*. Übrigens hat Schmiedeberg sein lebendiges Interesse an praktischen Fragen in der medizinischen Gesetzgebung, bei den Beratungen des Reichsgesundheitsamtes tuber das Arzneibuch oder über toxikologisch-hygienische Gegenstände (z. B. das nene Blei- und Zinkgesetz) mit seiner persönlichen, dureh Sachkunde und lauteres Urteil ansgezeichneten Betätigung nock bis in die allerletzte Zeit hinein bewiesen.

Schon vorher habe ich erwähnt, von wie großer Bedeutung es gewesen, daB Schmiedeberg gleich am Beginn seiner wissenschaftlichen Laufbahn in Dorpat mit B. Naunyn in Berührung und demnächst in enge wissenschaftliche und freundschaftliche Beziehung trat: gleich beim Beginn seiner Tätigkeit in Straßburg $1872 \mathrm{kam}$ es zur Gründung des von Klebs, Naunyn und Schmiedeberg herausgegebenen Archivs für experimentelle Pathologie und Pharmakologie, eines Unternelmens, das fur die Entwicklung der theoretischen Medizin in Deutschland von gröBter Bedeutung werden sollte. Durch die Vereinigung der Herausgeber und durch den Titel der Zeitschrift wurde die enge Beziehung der Pharmakologie zur Pathologie ansgedrückt, indem beide Wissenschaften sich in die gemeinsame Aufgabe zu teilen haben, die Lebenstorgänge unter abnormen Bedingungen za erforschen und zu beherrschen. Es hat dies in der Tat zu regem Austausch der Probleme und Arbeitsmethoden in den nahe verwandten Disziplinen gefïhrt und für alle Teile, insbesondere aber auch für die normale Physiologie reiche Früchte getragen: „Für das Endresultat ist es gleichguiltig, ob schließlich die Pathologie in die Pharmakologie aufgeht oder umgekehrt, und ob dam beide mit der Physiologie zu einer einheitlichen Lebenslehre zusammenfließen \%.

Da fuir die Sammlung pharmakologiseher Forschungen das Archiv lange Zeit das einzige war und bis zur Stunde noch eines der bedeutendsten geblieben ist, so war und ist auch der gröBere Anteil an den darin gebrachten Veröffentlichungen pharmakologisch und rechtfertigt in diesem Sinne die oft gebranchte kurze Bezeichnung als "Schmiedebergs Archiv*. 
Das in dem soeben aus dem *Grundriß a angeführten Satze enthaltene Programm kennzeichnet übrigens treffend Schmiedebergs eigene wissenschaftliche Vielseitigkeit. In erster Linie freilich betrachtete Schmiedeberg sich als bahnbrechenden Vorkämpfer und Vorarbeiter der Pharmakologie als solcher, und machte es sich zur Lebensaufgabe, dieser Disziplin die gesicherte wissenschaftliche Grundlage und Abgrenzung zu geben, und ihr dadurch den notwendigen and gebührenden Anteil an dem Unterricht und der Ausbildung der Ärzte, sowie die ihr angemessene Gleichstellung mit den Schwesterdisziplinen an den Universitäten zu erringen. In welchem Maße ihm dies Werk gelungen, braucht hier nicht ausgefuhrt zu werden. Schmiedebergs innerste persönliche Neigung gehörte aber doch nicht so sehr der experimentellpharmakologischen als der physiologischen, insbesondere der physiologisch- und pathologisch-chemischen Forschung; und ihr verdankt die Wissenschaft die reichen Frtichte seiner tiefgründigen Arbeiten über den normalen and den pathologisch oder pharmakologisch veränderten Stoffwechsel und den Chemismus der Gewebe.

Eine seiner ersten Arbeiten dieses Gebietes lieferte den Nachweis des Vorkommens von unterschwefliger Säure im Harn von Hunden und Katzen 1). Viel wichtiger und von grundsätzlicher Bedeutung war die in Gemeinschaft mit G. von Bunge durchgeführte Untersuchung über die Bildung der Hippursäure aus zugeführter Benzoësäure und Glykokoll im Organismus des Hundes²). Vorläufige Versuche an entleberten Fröschen hatten ergeben, daß die Leber nicht, oder zum wenigsten nicht allein, der Ort der Hippursäurebildung ist; an Hunden zeigte sich nun nach Unterbindung beider Nieren die Hippursäurebildung aufgehoben, und es gelang Schmiedeberg und Bunge in der ausgeschnittenen, mit defibriniertem Blut durchströmten Niere die Paarnng von Glykokoll und Benzoësäure nachzuweisen. Mit dem zerstampften und fein zerriebenen Nierenbrei gelang die Paarung unter keinen Umständen: es wurde hier zum ersten Male die grundsätzlich wichtige Tatsache gefunden und erkannt, daß die synthetischfermentative Leistung an lebendes Zellprotoplasma gebunden ist zum Unterschied von oxydativen und spaltenden Enzymen, als welche davon trennbar dargestellt werden können. Ein solches, in den Geweben weitverbreitetes spaltendes Ferment, das Histozy m, hat Schmiedeberg ans der Schweinsniere darzustellen gelehrt ${ }^{3}$ ), ein thermolabiles

1) Archiv für Heilkunde 1867, Bd. 8.

2) Dieses Archiv 1876, Bd. 6.

3) Ebenda 1881, Bd. 14. 
Oxydationsenzym wurde in ergänzenden Versuchen von Jaquety) aus abgetöteten Lungen und Nieren ausgezogen.

Mit diesen Versuchen ist die methodische Grundlage zu allen weiteren derartigen Untersnchungen über den Stoffwechsel in den Organen gegeben und bereits eine Reihe grundsätzlicher Fragen des Stoffwechsels ihrer Lösung zugeführt worden. Schon früher (1877) hatte Schmiedeberg eines der wichtigsten Stoffwechselprobleme in Angriff genommen, auf das ihn die bereits 1871 im Dorpater Institut gemachte Beobachtung gefuhrt hatte, daß der saure Harn der Fleischfresser selbst nach sehr reichlicher Zufuhr von kohlensaurem oder essigsaurem Ammon nicht wie nach entsprechender Fütternng mit Natriumkarbonat oder Azetat alkalische Reaktion annimmt. Schmiedeberg vermutete, daß das Ammoniumkarbonat in Harnstoff umgewandelt werde, und veranlaßte E. Hallervorden zu entsprechenden Versuchen, die in der Tat die erwartete Bestätigung brachten 2).

Ort und Bedingungen der Harnstoffeynthese mit Hilfe der Ludwigsehen Durchströmungsmethode an überlebenden Organen festzustellen, war nun die ron Schmiedeberg weiter ins Auge gefaßte Anfgabe: sie ist einige Jahre später bekanntlich in meisterhafter Weise durch W. von Schröder gelöst worden ${ }^{3}$.

In engen Zusammenhang mit dem Problem der Harnstoffbildung aus Ammonkarbonat trat die gleichzeitig in Angriff genommene Untersuchung tuber die Wirkung der Säuren auf den tierischen Organismus (Walter 1877). Es ergab sich, daß Pflanzenfresser leicht, Fleischfresser viel schwerer durch Säurezufuhr unter den Erscheinungen des Komas zu vergiften sind, und zwar weil letztere imstande sind, die zugeführte Säure zum Teil durch $\mathrm{NH}_{3}$ unter Ablenkung von der Harnstoffsynthese zu nentralisieren und so die Alkali karbonate des Blutes und der Gewebe zu schonen; die Pflanzenfresser besitzen diese Schutzorrichtung nicht, auch der Mensch nur in beschränktem Maße, so daß bei einer Säurevergiftung zwar auch viel Ammonsalz im Harn ausgeschieden wird, aber trotzdem ein so großer Verlust an Natriumkarbonat eintreten kann, daß der Vergiftete im Koma zugrunde geht. Dieser wichtige Fund hat dann, wie bekannt, zu der folgesehweren Entdeckung der Säurevergiftung durch Oxybuttersäure im diabetischen Koma und zu der oft lebensrettenden Behandlung durch Alkalieinlauf gefübrt (vgl. die Arbeiten der Na un yn-

1) Dieses Archiv 1892, Bd. 29.

2) Ebenda 1877, Bd. 8 .

3) Ebenda 1885, Bd. 19. 
schen Schule $\left.{ }^{1}\right)$ ). Auch bei einer großen Reihe anderer pathologischer Zustände, bei Infektionskrankheiten, bei vielen Vergiftangen, wie durch Phosphor, Arsen, Eisen u. a. m. ist die endogene Säurevergiftung, d. h. die Minderung der Karbonatalkaleszenz des Blutes eine wesentliche Begleiterscheinung und für das Verständnis der Symptome, der Prognose und der Therapie von Bedeutung.

Im Jahre 1876 hatte Schmiedeberg bei Gelegenheit einer Untersuchung über die Pharmakologie des Kampfers ${ }^{2}$ ) die Beobachtung gemacht, daß der verfütterte Kampfer im Harn nicht als solcher, sondern in einer nach dem Kochen mit Salzsäure CnO reduzierenden Verbindung ausgeschieden wird. Der nene Stoff ist dann 1879 von Schmiedeberg in Gemeinschaft mit mir rein gewonnen und analysiert worden: es ergab sich eine Verbindung des aus dem Kampfer oxydatir entstandenen Campherols mit einer reduzierenden Säure von der Formel $\mathrm{C}_{6} \mathrm{H}_{10} \mathrm{O}_{7}$, die mit Rücksicht auf ihre Verwandtschaft mit der Glukose den Namen Glukuronsäure erhielts). Mit der Glukuronsäure ist der Nachweis geliefert für die Möglichkeit des unmittelbaren oxydativen Abbaues der Glukose obne die sonst obligate vorangehende Spaltung in Milchsäure; spätere Untersuchungen Schmiedebergs haben dann dargetan, daß dieser Vorgang zu den normalen im Organismus gehört, insofern die Glukuronsäure sich als ein wesentlicher Bestandteil des Knorpelgewebes vorfindet, nämlich als Paarling in dem von Schmiedeberg ans dem Knorpel gewonnenen Chondrosin, einem Spaltprodukt der Chondroitinschwefelsäure 4$)$. Fuir die Beurteilung von Stoffwechselvorgängen bzw. Fäulnisprozessen im Organismus sind die gepaartẹn Glukuronsäuren von gröBter Bedeutung geworden; wahrscheinlich ist die Glukuronsäure auch als Quelle der Organpentosen anzusprechen.

Die schon in Dorpat 1869 unter Schmiédebergs Leitung and Teilnahme begonnene ${ }^{5}$ ) und später (1896-1898 durch Straub, Rosenstein und v. Vámossy) fortgeführte Untersuchung über den $\mathrm{CO}$ Diabetes hatte das auffällige Ergebnis gebracht, daß fuir das Zustandekommen des Diabetes nicht der Zuckervorrat im Organismus oder die Zuckerzufuhr entscheidend ist, sondern gewisse alkohollösliche Produkte der Eiweißrerdanung. Schmiedeberg hat sich

1) Coranda, Hallervorden, Dieses Arehiv 1879/80, Bd. 12.

2) Wiedemann, Ebenda 1876, Bd. 6.

3) Schmiedeberg a. H. Meyer, Hoppe Seylers Ztsehft. 1879, Bd. 3.

4) Schmiedeberg, Dieses Archiv 1891, Bd. 28.

5) L. Senff, Diss. Dorpat 1869. - Straub, Rosenstein, v. Vámossy, Dieses Arehiv Bd. 38, 40, 41 . 
mit diesem Gegenstand lange Jahre besehäftigt and schließlich es ist die letzte seiner Veröffentlichungen 1 ) - eine eigenartige Theorie des Diabetes mellitus daraus entwiekelt. Er schloß nämlich auf das Vorkommen einer » diabetogenen Substanz « im normalen Stoffwechsel, die jedoch nur beim Fehlen oder Versagen des regulierenden Pankreashormong wirksam werde, nämlich den Gewebszncker durch Anlagerung unangreifbar mache, bis er erst in der Niere wieder abgespalten tnd dann ungenutzt ausgeschieden werde. Danach wäre jede Art des Diabetes (CO, Adrenalin usw.) anf ungenügenden pankreatischen Schutz gegenüber der »diabetogenen Substanz * zu beziehen. - Schmiedeberg selbst hat im Schlußsatz die Möglichkeit mannigfacher Einwände zugegeben, und in der Tat läßt sich seine Theorie mit manchen neueren klinischen und experimentellen Beobachtungen einstweilen nicht in Einklang bringen. Darauf näher einzugehen ist hier wicht der Ort.

Von Schmiedebergs grundlegenden und umfassenden Arbeiten erwähne ich weiter die über viele Jahre sich erstreckende und anch erst ganz zuletzt in einer bedentsamen Abhandlung zusammengefaßten Untersuchungen über die stickstoffhaltigen Kohlehydratverbindungen der EiweiBstoffe ${ }^{2}$. Schmiedeberg gibt darin seine Darstellung und Analyse des Hyaloidins, d. i. des von ihm so benannten biuretfreien Mutterstoffes der in verschiedenen Eiweißkörpern enthaltenen CuO-reduzierenden Gruppen, erörtert die von ihm angenommene Strukturformel, wonach das Hyaloidin sich aus Diglukosamin, Hexose und Essigsäure zusammensetzt, und entwickelt die Beziehungen dieses Körpers zur Bildung der Chondroitinschwefelsäure, des Collagens und des Amyloids, sowie seine Verwandtschaft mit dem Chitin. - Die Weiterfübrung und Vollendung dieser für die Physiologie des Kohlehydrat- und Eiweißstoffwechsels ungemein wichtigen Untersuchungen ist durch das Abbrechen ron Schmiedebergs Tätigkeit in Straßburg verhindert worden.

Durch sein bei der Hyaloidindarstellang schon erfolgreich angewandtes "Kupferazetat-Verfahren * ist es Schmiedeberg auch gelungen zum erstenmal die Nakleinsäure analysenrein, d. h. ganz frei ron Eiweißspuren und von Protaminen zu gewinnen 3 ). Veranlassung und Material zu dieser Arbeit hatte Schmiedeberg aus dem Nachlaß Mieschers erhalten, dessen jahrelangen grundlegenden Untersuchungen über die Lachsmilch unvollendet und unveröffent-

1) Dieses Archir 1921, Bd. 90.

2) Ebenda 1920, Bd. 87.

3) Ebenda 1899, Bd. 43; 1907, Bd. 56; Nels on, Ebenda 1908, Bd. 59. 
licht geblieben waren und nun nach Mieschers Tode von Schmiedeberg vervollständigend bearbeitet und heransgegeben wurden.

In einer Ansprache in der Preußischen Akademie der Wissenschaften macht Planck gelegentlich die treffende Bemerkung > zu einer führenden Rolle in der Wissenschaft gehöre noch etwas anderes als selbst die allerreichste, durch vollendete Sachkenntnis geleitete and durch kritischen Scharfblick geziugelte Gestaltungskraft. Es gehöre dazu auch die Gewissenhaftigkeit, welche auch unscheinbaren Dingen, falls sie nur ein grundsätzliches Interesse beanspruchen, Beachtung schenkt und ihnen, wenn es sein muß, bis in kleine entlegene Winkel nachspürt. . «: ein solcher zähe und gewissenhafte Spürtrieb verrät sich bei Schmiedeberg fast noch deutlicher, als in den soeben angeführten großen und weitausschanenden Arbeiten, gerade in einer Reihe von feinen Einzeluntersuchungen, zunächst mehr deskriptiven Charakters, die zufälligen Anregungen entsprangen: so in der Aufindung einer Methode zur Reindarstellung der Paranußkrystalle ${ }^{1}$, die er als die Magnesiunverbindung des Vitellins erkannte; in der Darstellung und genauen Untersuchung des Sinistrins ${ }^{2}$ ), eines dextrinähnlichen, aber linksdrehenden Kohlehydrates aus der Meerzwiebel; endlich in der bewunderungswürdigen Analyse der Wohnröluren des Ringelwurmes Onuphis tubicola, aus welchen aus im ganzen $32 \mathrm{~g}$ Robstoft - er das Onuphin ${ }^{3}$ ) gewann und als einen dem Hyalin der Echinococeusblasen nahe verwandten, koblehydrathaltiger Körper kennen lehrte.

Diese nur das Wichtigste berührenden Angaben mögen genügen, um ron Schmiedebergs Bedeutung und von seinen Verdiensten auch auf dem Gebiet der physiologischen und pathologischen Chemie einen Begriff zu geben. - Schmiedebergs fachwissenschaftliche Arbeiten zu lesen ist nicht leicht, sein Stil ist bei aller Klarbeit und Reinheit der Form doch ungemein streng; die Darstellung sachlich gedrängt - genau genommen wohl kein Wort zu wenig, aber sicherlich auch keines zu viel; so daß der Leser zu angespannter Aufmerksamkeit und Sammlung genötigt wird. Das gilt ebenso von seinen einzelnen Abhandlungen, wie namentlich von seinem $\gg$ Grundriß «. Dagegen waßte er in populären Schriften, wie z. B. in dem Büchlein *Arzneimittel und GenuBmittel «4), auch eine allgemein verständliche Darstellung zu geben und oft auch einen anmutigen und

1) Hoppe Seylers Ztsehft. 1877, Bd. 1.

2) Ebenda 1879, Bd. 3.

3) Mitt. Zool. Stat. Neapel 1882, Bd. 3.

4) Leipzig, Teubner, 1912. 
selbst vergnüglichen Ton anzuschlagen: so in den $»$ Historischen and experimentellen Untersuchungen über die Zichorie and den Zichorienkaffee in diätetischer und gesundheitlicher Beziehnng*1), worin er sich für die arg verleumdete Zichorienwarzel einsetzt als einen "bei richtiger Zubereitung sehr wohlsehmeckenden und gesundheitlichen Ersatz des Kaffeess. Oder in der Studie „Über Naturwein und Kunstwein "2). Sebmiedeberg selbst wabte nicht nur einen edlen Wein zu schätzen, sondern hatte sich anch eine feine Kennerschaft in Weinfragen erworben. So spricht er mit offenbarer Frende von der harmonischen Rundung: and Vollkommenheit der Edelweine vom Rhein, von der Mosel, von Burgund und aus dem Bordeauxlande"; * Der Weinkenner wird nicht nur diese Gruppen von Edelweinen nicht verwechseln, sondern auch recht gut zu unterscheiden vermögen, welchen Anteil an dem Bouquet einerseits die Traubensorten und andererseits die Edelfänle und die alkoholische Gärung haben *. Und mit gebührender Verachtung wendet er sich zum Schutz der Edelweinzucht gegen alle durch Nachhilfe, wie das Gallisieren, verbesserten Sauerweine und gar gegen die "Kunstweine" schlechtweg: "Die Kunst der Weinbereitung besteht in der richtigen Leitung der Gärung und der passenden \Kellerbehandlong*. Der dabei erzielte Wein ist aber kein Kunstwein. Die Herstellung des letzteren erfordert keine Kunst.« "In einem solchen Gemisch schmeckt man jeden einzelnen Bestandteil, also vor allem Wasser, Alkohol, Säure nnd die verscbiedenen Bonquetstoffe, deutlich gesondert durch. In einem guten Wein wirken diese Stoffe weit weniger jeder für sich, sondern alle zusammen fast wie ein einheitlicher Stoff, und erzengen einen zwar nicht einfachen, aber doch harmonisch gerundeten Geschmackseindruck. Für die erfreulichen, aber auch für die nnter Umständen schädlichen WirKangen der Edelweine kommen neben dem Alkohol namentlich die jedem Wein eigenen Bouquetstoffe $*$ in Betracht: sie verursachen jene Erschlaffung der Hirngefäße, von der das Kopfweh abhängt, das dem unvorsichtigen Genusse zu folgen pflegt; manche Weine haben wegen der besonderen Beschaffenheit ihrer Bouquetstoffe diese Wirkung in hohem Grade und können daher für Kranke schädlich sein. »Eigentlich müßte jeder Arzt die Weine in dieser und jeder andern gesundheitlichen Beziehung kennen. Diese Kenntnis zu vermitteln ist eine der Aufgaben der Pharmakologie, die aber nock

1) Arch. f. Hygiene 1912, Bd. 74.

2) Über Naturwein und Kunstwein. Leipzig, Vogel, 1900. 
nicht an allen deutschen Universitäten in ausreichender Weise selbständig vertreten ist." Heute, nach 20 Jahren, wäre die gewünschte »ausreichende Vertretung " allenfalls vorhanden.

Schmiedeberg hatte einen empfänglichen Sinn für Geruchsund Geschmacksgenüsse, aber die höhere Befriedigung gewährte ihm dabei doch das feine verstandesmäßige Unterscheidungs- und $\mathbf{E r}-$ kenntnisvermögen, das in seiner Anlage vorhanden bewußt getibt and ausgebildet worden. Auch in seinem Verhältnis zur Kunst, namentlich der Architektur und Malerei, fur welche Schmiedeberg seit seiner fruhen Jugendzeit lebhaftes und tiefes Interesse bekundete, vereinigte sich die unmittelbare Freude des Genusses mit der grïndlichen Kennerschaft, die er sich durch oft wiederholte eingehende Studien in den Kunststätten Italiens und Spaniens erworben hatte Auf der guten und immer wieder auch für alle Naturwissenschaft bewährten Grundlage humanistischer Schulung und Erziehung fußend hatte Schmiedeberg sich auch die Hochschätzung und das Verständnis der alten Sprachen und ihres Schrifttums bewahrt. In seinen Arbeiten finden sich mannigfache Belege seiner geschichtlichen und sprachlichen Quellenstudien, und noch als Achtzigjähriger hat Schmiedeberg in den Schriften der Wissenschaftlichen Gesellschaft in Straßburg (1918) eine eingehend gelehrte und kritische, historisch und sachlich ebenso lehrreiche wie anziehende Abhandlung über die Pharmaka in der Ilias und Odyssee veröffentlicht.

In der Vorlesung für Studierende gab Schmiedeberg seine Lehre mit großem Ernst, mehr dogmatisch als diskutierend, stets in freier, genau durchdachter Rede; der Vortrag war wie sein Stil nüchtern, gedrungen, sehr inhaltreich und von tiberlegenem und sehr bestimmt gefaßtem Urteil; er war deshalb trotz des Verzichtes auf allen Redeschmuck und Glanz immer höchst eindrucksvoll und von nachhaltiger Wirkung. Bei der Erörterung einer wissenschaftlichen oder auch politischen Frage im Gespräch ließ Schmiedeberg sich meiner Erinnerung nach wenig auf weitläufige Widerlegung: entgegenstehender Ansichten oder Einwürfe ein, sondern gab in einigen lapidaren Sätzen seiner wohlerwogenen und festgehaltenen Meinung entschiedenen Ausdruck. Es war nicht seine Sache und auch nicht seine Absicht auf fremde Gedankengänge einzugehen in dieser Einseitigkeit, um nicht zu sagen Starrheit, lag mit ein Teil seiner zielbewußten Kraft und auch seines Erfolges. Im tabrigen ließ er den Schülern im Laboratorium, so weit sie schon selbständig zu arbeiten imstande waren, freie Hand, bekümmerte sich jedoch genan um eines jeden Arbeit in Anlage, Ausfübrung und kritischer Ver- 
wertung. Mit Anfängern konnte Schmiedeberg sich methodisch sehr eingehend und persönlich beschäftigen und sie in allen Kunstregeln und Handgriffen selbst unterweisen: das Ziel war stets, zum selbständigen Denken und Urteilen und zu genanem, vor allem aber zu streng gewissenhaftem Arbeiten zu erziehen.

Unerreicht als balnbrechender und schöpferischer Geist ist uns der Meister in seinem nnermtidichen Fleik, seiner tiefen, auch das Kleinste würdigenden Gewissenhaftigkeit und in der grîndlichen Verachtung allen Scheines und aller kleinlichen Eitelkeit - ist Schmiedeberg in seiner schlichten, einfachen Wirklichkeit uns allen, die wir unter seinen Augen zu arbeiten das Glück hatten, ein Ehrfurcht und Nachfolge gebietendes Vorbild geblieben.

Dem in Schmiedebergs Festbande dieses Archirs 1908 entbaltenen Verzeichnis seiner Arbeiten sind hinzuzuftigen:

Rudolf Buchheim, Nachruf. Dieses Archiv 1912, Bd. 67. - Historische und experimentelle Untersuchungen über die Zichorie usw. Archiv f. Hygiene 1912, Bd. 74. - Untersuchnngen über die chemische Zusammensetzung der Wandung der Echinococcusblasen usw. Madelnngs Festschrift, Tübingen 1916. Über: elastische Verkïrzung nnd aktive Erschlaffung lebender Muskeln. Dieses Archiv 1917, Bd. 82. - Die Pharmaka der llias und Odyssee. Schriften der wissenschaftl. Ges. in StraBbarg 1918. - Über die Bestimmung des Wirknngswertes der Digitalis usw. Dieses Archiv 1919, Bd. 62. - Über die stickstoffhaltigen Kohlehydratverbindungen der Eiweißstoffe. Über die Kohlehydratabkömmilinge der Mukoide und Mncine, Über die Beziehungen des Hyaloidins zur Bildung der Chondroitinschwefelsäure, des Collagens und des Amyloíds im Organismas. Über Chitin and Chitinabkömmlinge des Tier- und Pfanzenreiches. Dieses Archiv 1920, Bd. 87, Heft 1/2. - Über die Vorgänge bei der Zuckerausscheidung im Diabetes. Dieses Archiv 1921, Bd. 90. 